\title{
Tunable Stimuli-Responsive Polymers for Cell Sheet Engineering
}

\author{
Nithya Joseph, Anil Kumar P R and TV Kumary \\ Division of Implant Biology, Biomedical Technology Wing \\ Sree Chitra Tirunal Institute for Medical Sciences and Technology \\ India
}

\section{Introduction}

Anchorage dependent cells require a suitable substrate or scaffold for attachment, adhesion and growth. Conventionally cells are detached from substrates by mechanical or enzyme treatment, which can affect cell re-adhesion, viability etc. Poly (N-isopropylacrylamide) [PNIPAAm] is a well known temperature sensitive polymer with LCST of $32^{\circ} \mathrm{C}$ and serve as a good substrate facilitating cell detachment without the use of proteolytic enzymes. Polymer and copolymers with $\mathrm{N}$-isopropylacrylamide [NIPAAm] been proved to be excellent for cell detachment by temperature treatment. The co-monomers provide functional groups that bind to biological molecules and form hybrid polymer for enhanced cell function. This chapter reviews and compares different smart intelligent surfaces, method of preparation, characterization techniques and applications of cell sheet engineering. PNIPAAm based surfaces are able support cell growth and then to detach without any chemical reagent step. These thermo responsive substrates avoid the problem associated with scaffold based tissue engineering.

\section{Cell sheet engineering}

Tissue engineering involves ex vivo expansion of anchorage dependent cells in three dimensions to provide functional tissues (Langer and Vacanti, 1993). Prof. Robert Langer, Dr. Joseph and Dr. Charles Vacanti proposed the concept of tissue engineering in order to overcome the limitations of organ transplantation. Over the past 20 years, using the technology of tissue engineering, many kinds of tissues such as bone, heart valves, cartilage, corneal epithelium, cardiac muscles and skin have been successfully reconstructed (Yang et al, 2005; Nishida et al, 2004). Most anchorage dependent mammalian cells must adhere to a solid substrate in order to proliferate and manifest their function. The substrates used for cell growth should possess many features like the ability to encourage cell adhesion, spreading and proliferation. Also it is essential that the cells are easily detached from the substrate after it has grown. Conventionally enzymatic proteolysis of extracellular matrix (ECM) with trypsin or other proteolytic enzymes used to detach cells from substrates which can affect cell re-adhesion, cell viability etc (Kumashiro et al, 2010). Thermoresponsive polymers are of great interest in tissue engineering applications specifically in cell sheet detachment. The effect of these stimuli responsive polymers was first studied by Heskins 
and Guillet in early 1960's (Heskins and Guillet, 1968). The smart behavior of these polymers is because of its change in molecular conformation in response to a temperature change in the surrounding environment. N-isopropylacrylamide [NIPAAm] is a well characterized thermo responsive polymer that shifts its phase properties above and below its lower critical solution temperature (LCST) of $32{ }^{\circ} \mathrm{C}$. Above the LCST, polymer will be hydrophobic resulting in the attachment and growth of cells while below the LCST it turns hydrophilic enabling the detachment of cells as a single cell or as a uniform cell sheet. Since the cell sheet could keep the cell junctions and deposited extra cellular matrix along with it, physiological functions and bioactivity of the cell sheet are similar to the cells in tissue, which could provide potential application as an engineered tissue. Polymer and copolymers with NIPAAm has been proved to be excellent for cell detachment by temperature variation. The group of Okano and coworkers done a lot of studies in thermoresponsive, Poly(NIPPAm) based polymers and proposed a new approach in tissue engineering called cell sheet engineering (Yamada et al, 1990).

\section{Mechanism of cell attachment and detachment from the thermoresponsive surfaces}

Cells initially attach to a surface by a process called passive adhesion in which the adherence is due to the physicochemical interaction such as hydrophobic interactions, coulomb forces and van der Waal's forces. Then the cells bind to a specific site on adhesive protein by a process called active adhesion via a receptor protein called integrin. Conventionally enzymes are used to detach cells from substrates, resulting in the destruction of cell membrane proteins and ECM which can affect cell re-adhesion and viability. But cell detachment from thermoresponsive surfaces retain their cellular structure and functions in which cell detachment occurs in a two step mechanism via a passive step, followed by an active step. In the passive step cell detachment is induced by the hydration of PNIPAAm chains on the substrate whereas in active step change of cell shape and detachment from the surface is driven by cytoskeletal action and metabolic process (Okano et al, 1995).

\section{Methods of preparation of temperature responsive intelligent surfaces}

Thermoresponsive polymers can be grafted on different substrates such as silicon, glass, quartz, polyethyleneterephthalate sheets etc but commonly grafted on Tissue Culture grade Poly Styrene (TCPS). The choice of substrate, method of application of thermoresponsive polymer on the substrate and thickness of the grafted polymer has a major role on cell attachment and detachment phenomena (Elloumi-Hannachi et al, 2010). It is reported that a grafting thickness of 15 to $20 \mathrm{~nm}$ is a prerequisite for efficient cell attachment and detachment (Akiyama et al, 2004).

Many types of polymerization techniques can be used for grafting the thermoresponsive surfaces such as Electron beam irradiation, Gamma radiation, Plasma polymerization, UV irradiation and Atom transfer radical polymerization.

\subsection{Electron beam induced polymerization}

Electron beam polymerization (EB) is a popular method used for producing thermoresponsive surfaces for cell sheet engineering (Yamato et alj, 2010). NIPAAm monomer is covalently bonded onto the TCPS (tissue culture polystyrene) dishes by the irradiation of electron beam 
on to the monomer. Electron beam method facilitates even, thin grafting and the large scale production of temperature responsive culture dishes even though it is expensive. The thickness is controlled by monomer concentration and radiation energy (Nagase et al, 2009).

\subsection{Gamma irradiation}

Gamma radiation is another method for the grafting of thermoresponsive surfaces on TCPS (Akiyama et al, 2004; Anil Kumar et al, 2007). This study also revealed that grafting by gamma irradiation is comparable with conventional electron beam irradiation and facilitates batch processing of polymerization and grafting of PNIPAAm. Moreover it avoids most sophisticated equipment like electron beam accelerator. The efficacy of the grafted surface was confirmed by the successful growth of different cell lines such as L-929 (mouse subcutaneous connective tissue fibroblast), NRK-49F (normal rat kidney), SIRC (rabbit corneal epithelium), and HOS (human osteosarcoma) and detachment of the cell sheets while maintaining the cell-cell and cell-extra cellular matrix contact.

\subsection{Plasma polymerisatiom}

Plasma polymerization is a different single step method which facilitates the preparation of thermoresponsive coatings on a solid substrate. A plasma glow discharge of NIPAAm monomer vapour was used to deposit PNIPAAm onto solid surfaces such as silicon, glass or TCPS. The cell adhesion/detachment studies showed to be insensitive to the grafted layer thickness. But this method is not suitable for large scale production due to the difficulties related with continuous treatment and size (Canavan et al, 2005).

\subsection{UV irradiation}

UV irradiation is a yet another approach for the grafting of thermoresponsive coatings on to the solid substrate. Photopolymerisation and photografting of PNIPAAm is done on TCPS by UV irradiation ( $365 \mathrm{~nm}$ for 5 to $30 \mathrm{~min}$ ) using photo initiators such as benzophenone (Nagase et al, 2009).

\subsection{Atom transfer radical polymerization technique (ATRP)}

ATRP facilitates the preparation of surfaces with dense polymer brushes from surface immobilized ATRP initiators (Mizutani et al, 2008). Preparation of PNIPAAm brushes on poly(4 vinyl benzyl chloride) coated TCPS surface using ATRP and application of these grafted surfaces to thermoresponsive cell culture substrates were studied by Mizutami etal. Attachment/ detachment process is favorable only in thinner surface less than $30 \mathrm{~nm}$.

\subsection{Reversible addition fragmentation chain transfer polymerization technique (RAFT)} RAFT polymerization technique allows the preparation of thermoresponsive PNIPAAm brushes with controlled chain length and graft density for cell sheet harvest. Chain length and density of PNIPAAm grafted on the surfaces have a significant effect on the thermoresponsive cellular behavior.

\subsection{Oxygen plasma treated PIPAAm surface}

Oxygen plasma treated PNIPAAm film is a promising material as a cell culture substrate in cell sheet engineering (Shimizu et al, 2010).A cast and dried film of thermoresponsive 
PNIPAAm was fabricated and treated with high intensity oxygen plasma. This novel method of cell sheet has mechanical strength with high stability and low cost.

\subsection{Solution casting method}

Most of the above techniques are expensive and might not be reachable to all researchers. An auxiliary simple and cost effective approach is the coating of thermoreponsive polymer onto the TCPS by solution casting method. Thickness of coating obtained by this method is reported to be in micron level and astonishingly doesn't affect the cell attachment and proved to be efficient for cell sheet attachment and detachment process (Joseph et al, 2010; Varghese et al, 2010).

\section{Different methods of characterization of thermoresponsive substrates}

Different characterization techniques are employed for the physicochemical and biological evaluation of thermoresponsive substrates which is very important. Physicochemical characterization techniques such as Attenuated total reflectance Fourier Transform spectroscopy (ATR-FTIR), Nuclear magnetic Resonance spectroscopy (NMR) are used for the qualitative and quantitative detection, while Atomic Force Microscopy (AFM), Ellipsometry, surface Plasmon resonance, Profilometry are used for the determination of thickness of the grafting or coating.

The presence and quantity of PNIPAAm on the thermoresponsive surface can be determined by the technique ATR-FTIR (Akiyama et al, 2007). The presence of characteristic peak of amide carbonyl group around $1650 \mathrm{~cm}^{-1}$ showed the presence of NIPAAm. The amount of PNIPAAm on the surface of TCPS significantly affects cell adhesion behaviour. The quantity of grafted PNIPAAm could be detected by the ratio of peak intensities $\left(\mathrm{I}_{1650} / \mathrm{I}_{1600}\right)$ in ATR-FTIR.

NMR technology also provides quantitative as well as qualitative assessment of thermoresponsive surface (Joseph et al, 2010).This techniques provides detailed structural information about the polymers and gives a better understanding about the polymerization mechanism.

Water contact angle measures the hydrophobicity and hydrophilicity of the surface and a contact angle of $70^{\circ}$ proved to be optimal for cell sheet adhesion and detachment (da Silva et al, 2007). The thermoresponsive surface wettability changes of free end linear and multipoint attached PNIPAAm surfaces were investigated using the Wilhelmy plate technique. A large contact angle change was observed for an end grafted PNIPAAm surfaces whereas a small contact angle change for multipoint attached surfaces due to the restricted chain conformation of the multipoint attached grafted surfaces.

Differential scanning calorimetry (DSC) is widely used for the detection of LCST. The LCST of homopolymer PNIPAAm lies around $32{ }^{\circ} \mathrm{C}$ and copolymers of PNIPAAm centered below or above $32^{\circ} \mathrm{C}$ depending upon the hydrophobicity and hydrophilicity of the comonomer.

Xray Photoelectron Spectroscopy (XPS) is one of the efficient methods for the qualitative assessment of the presence of PNIPAAm on different substrates (Jun and Okano, 2010)The elemental composition of carbon, nitrogen and oxygen on the grafted thermoresponsive surface, could be determined and should be in good agreement with the values based on the stochiometry of the monomer, NIPAAm. 
AFM can be used for the quantitative and qualitative characterization of thermoresponsive surfaces such as to observe the nano texture of the surface and to measure surface roughness and the thickness of the PNIPAAm coating (Jun and Okano, 2010). Profilometry is another method to visualize the surface morphology and the quantitative analysis of surface roughness and thickness from the surface profile measurement (Joseph et al, 2010). Determination of thickness from ellipsometry and surface plasmon resonance is very difficult because of the similarity in refractive index of both PNIPAAm and polymeric substrates (Nagase et al, 2009).

Biological evaluations of thermoresponsive surface is the most important characterization technique for cell sheet engineering and are done by cytotoxicity analysis, cell activity measurement, thermoresponsive efficacy, viability analysis and cell sheet characterization etc. The cytocompatibility, specific cytocompatibility, thermoresponsive efficacy studies are usually done using L929 cells, SIRC cells, HOS, NRK 49 F,Human fetal lung fibroblasts (TIG1), primary cells from rabbit cornea, rat primary hepatocytes etc (Anil Kumar et al, 2007; Joseph et al, 2010; Varghese et al, 2010). Cells were maintained in MEM (Minimum essential medium) supplemented with 10\% FBS (fetal bovine serum), $100 \mathrm{IU} / \mathrm{mL}$ pencilin and 100 $\mu \mathrm{g} / \mathrm{ml}$ streptomycin at $37^{\circ} \mathrm{C}$ in a $95 \%$ humidified atmosphere with $5 \% \mathrm{CO}_{2}$. The cytotoxicity analysis can be done by using direct contact method, indirect contact method;.High density polyethylene and zinc diethyl dithiocarbamate stabilized polyvinylchloride discs are usually used as negative and positive controls respectively. The cytotoxic evaluation is done by the comparison of the morphology of cells with positive and negative controls. MTT \{(3-(4,5-dimethyl thiazol -2-yl) -2,5-diphenyl tetrazolium bromide $\}$ cell culture assay is usually used to evaluate the cell proliferation. Absorbance is measured using a multiwell plate reader at $540 \mathrm{~nm}$. Cell viability is usually determined using neutral red staining. The thermoresponsive efficacy of the substrate is evaluated by retrieving the cell sheets by lowering the temperature below the LCST of the polymer and the viability of the retrieved cell sheet is determined using FDA (fluorescein diacetate) staining and observed under a fluorescence microscope. The cell sheet characterization is done by immuno staining technique using antibodies against proteins characteristic to tissue of interest.

\section{Various thermoresponsive substrates for cell sheet engineering}

Thermoresponsive substrates designed for cell sheet engineering have mainly used PIPAAm and its copolymers for cell adhesion and detachment. The use of thermoresponsive substrate to detach the confluent cell sheets without the use of conventional enzymatic treatments was first reported by Takezawa et al in 1990 (Takezawa et al, 1990). In this study they have used PIPAAm as a substratum by conjugating it with collagen for the culture of human dermal fibroblasts. These fibroblasts monolayer were harvested at reduced temperature by the dissolution of the dish coating. By the same time Yamado et al reported the successful culture of bovine hepatocytes on PNIPAAm grafted TCPS (Yamada et al, 1990). Homopolymer of NIPAAm has been reported to be grafted by various methods such as Electron beam polymerization, UV irradiation, Gamma irradiation etc and proved to be an excellent substrate for cell sheet engineering.

Copolymerization of NIPAAm with hydrophobic or hydrophilic monomer can modulate LCST for the systematic regulation of cell attachment and detachment. Thermoresponsive 
copolymers based on N-isopropylacrylamide-Co-glycidylmethacrylate(NGMA), Nisopropylacrylamide-methylmethacrylate (NMMA), (Varghese et al, 2010) Nisopropylacrylamide-butylmethacrylate (Tsuda et al, 2004) and NIPAAm-MMAphosphorylated HEMA (Thomas et al, 2010) were successfully used as a substrate for cell sheet harvesting. Thermoresponsive copolymer NGMA was clearly identified as potential substrate for cell culture harvesting system for generating 3D synthetic tissue. NGMA was synthesized by solution polymerization followed by the coating on TCPS surfaces by solution casting method. Copolymerisation of PIPAAm with the hydrophobic monomer glycidylmethacrylate results in a decrease of LCST. Thermoresponsive copolymer NMMA was also reported to be synthesized and coated by the same methodology mentioned above and proved to be an ideal thermoresponsive substrate with good cytocompatibility for cell culture and tissue reconstruction. Hydrophobic monomer $n$ butylmethacrylate (BMA) has been copolymerized with PNIPAAm to reduce the LCST of NBMA inorder to modulate the cell adhesion/detachment from culture dishes and revealed to be an efficient substrate for cell sheet engineering. Poly (N-vinylcaprolactum-co- N- isopropylacrylamide) PNVCL-coPNIPAAm grafted on TCPS dishes by EB irradiation were efficiently applied to detach viable cell sheets from culture surfaces (Lim et al, 2007).

The spontaneous recovery of cell sheet is important for maintaining the viability of retrieved cell sheets. PNIPAAm grafted onto porous membranes facilitate the acceleration of cell sheet detachment by providing rapid water movement between the interface of cell sheets and membrane surfaces (Kwon et al, 2000a). Another method for the acceleration of cell sheet detachment is by cografting of a hydrophilic polymer Polyethylene glycol (PEG) with the thermoresponsive polymer PNIPAAm (Kwon et al, 2000b). Copolymerisation with 2carboxyisopropylacrylamide (CIPAAm), $\{$ P(IPAAm-co-CIPAAm) $\}$ grafted surface accelerates the cell detachment process because of the presence of hydrophilic carboxyl group (Ebara et al, 2003).

\section{Biomolecule immobilizing thermoresponsive substrates}

Biomolecule immobilizing thermoresponsive cell culture dishes are known to be the next generation thermoresponsive substrates in cell sheet engineering (Jun and Okano, 2010). Regulation of cellular metabolisms and functions in culture are important aspects in cell sheet engineering. The simple approach to enhance the cellular activities are by immobilization or coating of bioactive compounds such as collagen, fibrin, elastin and arginine-glycine-aspartic acid (RGD) containing peptides on the cell culture substrate (Ebara et al, 2004). Anchoring of these bioactive compounds to this thermoresponsive substrate through chemical bonds is expected to increase the stability of the modified surface to a prolonged period. The interactions of these biomolecule ligands and cell membrane receptors have an important role in cell spreading, proliferation, differentiation, and signal transduction. This can be achieved either through coating or by the use of coupling agents or else by copolymerizing with an analogue of Isopropylacrylamide.

Cell adherence and growth on the thermoresponsive substrate is improved by coating cell adhesion promoters such as collagen, laminin and fibrin (Moran et al, 2007). But repeated washing will remove the coating of biomolecules. Immobilization is the process which helps to increase the stability and make possible their repeated or continued use.

Copolymerizing with an analogue of isopropylacrylamide, 2-carboxyisopropylacrylamide (CIPAAm) which has a carboxylate side chain that favours the immobilization of bioactive 
molecules onto thermoresponsive substrates. The synthetic cell adhesive peptide Arg-GlyAsp (RGD) found in extracellular matrix proteins has been successfully immobilized onto the grafted polymer chains via the above mentioned method using the coupling agents 1-Ethyl-3-[3-dimethylaminopropyl]carbodiimide hydrochloride [EDC]. The cells adhered and spread well on these surface even in the absence of serum. Another method of immobilization is by the coupling of biotinylated biomolecules onto streptavidin immobilized thermoresponsive substrate by affinity binding without any coupling agents. The co-immobilisation of cell adhesive peptide RGDs and cell growth factor insulin facilitated the cell adhesion and cell proliferation respectively.

The epoxy ring opening of GMA moiety is well known for incorporating diverse type of biomolecules. The uniqueness of the thermoresponsive substrate such as $\mathrm{N}$-isopropylacrylamide-co-glycidylmethacrylate is that, it facilitates the immobilization of biomolecules without any coupling agents by the presence of unreacted epoxy rings.

The biomolecule immobilized surfaces facilitates cell adhesion, culture and harvest of cell sheets which enables serum free cell culture and enzyme free cell harvesting.

\section{Supports for the transfer of cell sheet}

A cell sheet carrier is a support which facilitates the transfer of retrieved cell sheets. An ideal material as a cell sheet carrier should possess the features like biocompatibility, surface pliability, mechanical strength, hydrophilicity, presence of surface reactive groups which enable easy modification etc. Polyvinylidene fluoride membranes in the form of annular ring, fibrin gel coated membranes are some of the systems which have been used till today. Polyion complex gels are further developed as cell sheet carriers (Tang et al, 2007) but possess limited mechanical strength and have application difficulties. A further innovative development in this field is the cell culture substrate with combined properties of thermoresponsiveness and cell sheet carrier tool. Modified over head projection transparency sheet based on polyethyleneterephthalate coated with thermoresponsive $\mathrm{N}$-isopropylacrylamide-Co-glycidylmethacrylate is reported to be an efficient cell culture substrate as well as a cell sheet carrier tool (Joseph et al, 2010). The study demonstrated that a simple modification of commercially available PET sheets by hydrolysis followed by coating of thermoresponsive NGMA forms a biocompatible substrate for scaffold free cell sheets as well as a cell sheet carrier tool.

\section{Clinical applications of cell sheet engineering}

Cell sheet engineering favours the formation of scaffold free transplantable three dimensional tissues from thermoresponsive cell culture substrate. This technology has been used in corneal surface reconstruction, myocardial tissue reconstruction, regeneration of damaged periodontal tissue, treatment of oesophageal ulceration, treatment for type 1 diabetes etc (Elloumi-Hannachi et al, 2010). Some of the clinical applications reported till dates are reported below. This technology in corneal surface reconstruction has been successfully applied to the patients suffering from unilateral or bilateral corneal stem cell deficiencies due to alkali burns or Stevens Johnson syndrome. These reconstructed corneal surfaces are able to restore the vision. Cell sheet technology for the treatment of oesophageal ulceration enhances the wound healing and reduces the host inflammatory responses. This is reported to be the first clinical case in the world which is applied to endoscopy with 
successful wound healing and improved post operative quality of life. Transplantation using cell sheets derived from periodontal ligament is reported to be useful for the regeneration of damaged periodontal tissue. Cell sheet technology using pancreatic islets has been used for the treatment of type1 diabetes. Another clinical application of cell sheet engineering is its use in the treatment for dilated cardiomyopathy and cardiovascular tissue repair by myocardial tissue reconstruction. This smart culture surfaces have already been used in clinical trials and thus it offers a new frontier in the biomedical field.

\section{References}

Abraham, T. N., V. Raj, T. Prasad, P. R. Anil Kumar, K. Sreenivasan and T. V. Kumary (2010). A novel thermoresponsive graft copolymer containing phosphorylated HEMA for generating detachable cell layers. Journal of Applied Polymer Science 115(1): 52-62.

Akiyama, Y., A. Kikuchi, M. Yamato and T. Okano (2004). Ultrathin poly(Nisopropylacrylamide) grafted layer on polystyrene surfaces for cell adhesion/detachment control. Langmuir 20(13): 5506-5511.

Akiyama, Y., A. Kushida, M. Yamato, A. Kikuchi and T. Okano (2007). Surface characterization of poly(N-isopropylacrylamide) grafted tissue culture polystyrene by electron beam irradiation, using atomic force microscopy, and X-ray photoelectron spectroscopy. J Nanosci Nanotechnol 7(3): 796-802.

Anil Kumar, P. R., K. Sreenivasan and T. V. Kumary (2007). Alternate method for grafting thermoresponsive polymer for transferring in vitro cell sheet structures. Journal of Applied Polymer Science 105(4): 2245-2251.

Canavan, H. E., X. Cheng, D. J. Graham, B. D. Ratner and D. G. Castner (2005). Cell sheet detachment affects the extracellular matrix: a surface science study comparing thermal liftoff, enzymatic, and mechanical methods. J Biomed Mater Res A 75(1): 113.

da Silva, R. M., J. F. Mano and R. L. Reis (2007). Smart thermoresponsive coatings and surfaces for tissue engineering: switching cell-material boundaries. Trends Biotechnol 25(12): 577-583.

Ebara, M., M. Yamato, M. Hirose, T. Aoyagi, A. Kikuchi, K. Sakai and T. Okano (2003). Copolymerization of 2-carboxyisopropylacrylamide with $\mathrm{N}$-isopropylacrylamide accelerates cell detachment from grafted surfaces by reducing temperature. Biomacromolecules 4(2): 344-349.

Ebara, M., M. Yamato, T. Aoyagi, A. Kikuchi, K. Sakai and T. Okano (2004). Immobilization of cell-adhesive peptides to temperature-responsive surfaces facilitates both serumfree cell adhesion and noninvasive cell harvest. Tissue Eng 10(7-8): 1125-1135.

Elloumi-Hannachi, I., M. Yamato and T. Okano (2010). Cell sheet engineering: a unique nanotechnology for scaffold-free tissue reconstruction with clinical applications in regenerative medicine. J Intern Med 267(1): 54-70.

Heskins, M. and J. E. Guillet (1968). Solution Properties of Poly(N-isopropylacrylamide). J. Macromol. Sci. Chem. 2(8): 1441-1455.

Hyeong Kwon, O., A. Kikuchi, M. Yamato and T. Okano (2003). Accelerated cell sheet recovery by co-grafting of PEG with PIPAAm onto porous cell culture membranes. Biomaterials 24(7): 1223-1232. 
Joseph, N., Anil Kumar P.R., T. Prasad, L. Joseph, S. K and K. T.V. (2010). Intelligent thermoresponsive substrate from modified overhead projection sheet as a tool for construction and support of cell sheets in vitro. Tissue Engineering: Part C 17(2): 181191.

Joseph, N., T. Prasad, V. Raj, P. R. Anil Kumar, K. Sreenivasan and T. V. Kumary (2010). A Cytocompatible Poly(N-isopropylacrylamide-co-glycidylmethacrylate) Coated Surface as New Substrate for Corneal Tissue Engineering. Journal of Bioactive and Compatible Polymers 25(1): 58-74.

Jun, K. and O. Teruo (2010). Fabrication of a thermoresponsive cell culture dish: a key technology for cell sheet tissue engineering. Science and Technology of Advanced Materials 11(1): 014111.

Kumashiro, Y., M. Yamato and T. Okano (2010). Cell attachment-detachment control on temperature-responsive thin surfaces for novel tissue engineering. Ann Biomed Eng 38(6): 1977-1988.

Kwon, O. H., A. Kikuchi, M. Yamato, Y. Sakurai and T. Okano (2000). Rapid cell sheet detachment from poly(N-isopropylacrylamide)-grafted porous cell culture membranes. J Biomed Mater Res 50(1): 82-89.

Langer, R. and J. P. Vacanti (1993). Tissue engineering. Science 260(5110): 920-926.

Lim, Y. M., J. P. Jeun, J. H. Lee, Y. M. Lee and Y. C. Nho (2007). Cell Sheet Detachment from Poly(N-vinylcaprolactam-co-N-isopropylacrylamide) Grafted onto Tissue Culture Polystyrene Dishes. Journal of Industrial and Engineering Chemistry 13(1): 21-26.

Mizutani, A., A. Kikuchi, M. Yamato, H. Kanazawa and T. Okano (2008). Preparation of thermoresponsive polymer brush surfaces and their interaction with cells. Biomaterials 29(13): 2073-2081.

Moran, M. T., W. M. Carroll, I. Selezneva, A. Gorelov and Y. Rochev (2007). Cell growth and detachment from protein-coated PNIPAAm-based copolymers. J Biomed Mater Res A 81(4): 870-876.

Nagase, K., J. Kobayashi and T. Okano (2009). Temperature-responsive intelligent interfaces for biomolecular separation and cell sheet engineering. J R Soc Interface 6 Suppl 3: S293-309.

Nishida, K., M. Yamato, Y. Hayashida, K. Watanabe, K. Yamamoto, E. Adachi, S. Nagai, A. Kikuchi, N. Maeda, H. Watanabe, T. Okano and Y. Tano (2004). Corneal reconstruction with tissue-engineered cell sheets composed of autologous oral mucosal epithelium. N Engl J Med 351(12): 1187-1196.

Okano, T., N. Yamada, M. Okuhara, H. Sakai and Y. Sakurai (1995). Mechanism of cell detachment from temperature-modulated, hydrophilic-hydrophobic polymer surfaces. Biomaterials 16(4): 297-303.

Shimizu, K., H. Fujita and E. Nagamori (2010). Oxygen plasma-treated thermoresponsive polymer surfaces for cell sheet engineering. Biotechnol Bioeng 106(2): 303-310.

Takezawa, T., Y. Mori and K. Yoshizato (1990). Cell Culture on a Thermo-Responsive Polymer Surface. Nat Biotech 8(9): 854-856.

Tang, Z., A. Kikuchi, Y. Akiyama and T. Okano (2007). Novel cell sheet carriers using polyion complex gel modified membranes for tissue engineering technology for cell sheet manipulation and transplantation. Reactive and Functional Polymers 67(11): 1388-1397. 
Tsuda, Y., A. Kikuchi, M. Yamato, Y. Sakurai, M. Umezu and T. Okano (2004). Control of cell adhesion and detachment using temperature and thermoresponsive copolymer grafted culture surfaces. J Biomed Mater Res A 69(1): 70-78.

Varghese, V., V. Raj, K. Sreenivasan and T. Kumary (2010). In vitro cytocompatibility evaluation of a thermoresponsive NIPAAm-MMA copolymeric surface using L929 cells. Journal of Materials Science: Materials in Medicine 21(5): 1631-1639.

Yamada, N., T. Okano, H. Sakai, F. Karikusa, Y. Sawasaki and Y. Sakurai (1990). Thermoresponsive polymeric surfaces; control of attachment and detachment of cultured cells. Die Makromolekulare Chemie, Rapid Communications 11(11): 571-576.

Yamato, M., O. H. Kwon, M. Hirose, A. Kikuchi and T. Okano (2001). Novel patterned cell coculture utilizing thermally responsive grafted polymer surfaces. J Biomed Mater Res 55(1): 137-140.

Yang, J., M. Yamato, C. Kohno, A. Nishimoto, H. Sekine, F. Fukai and T. Okano (2005). Cell sheet engineering: recreating tissues without biodegradable scaffolds. Biomaterials 26(33): 6415-6422. 


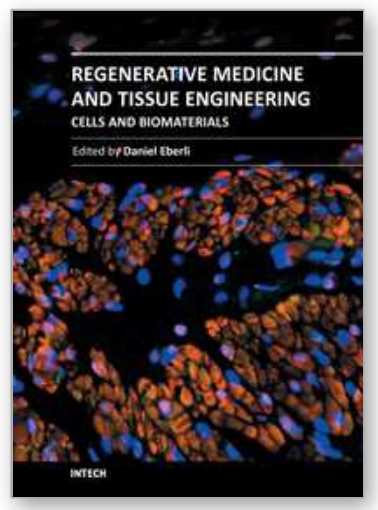

\author{
Regenerative Medicine and Tissue Engineering - Cells and \\ Biomaterials \\ Edited by Prof. Daniel Eberli
}

ISBN 978-953-307-663-8

Hard cover, 588 pages

Publisher InTech

Published online 29, August, 2011

Published in print edition August, 2011

Tissue Engineering may offer new treatment alternatives for organ replacement or repair deteriorated organs. Among the clinical applications of Tissue Engineering are the production of artificial skin for burn patients, tissue engineered trachea, cartilage for knee-replacement procedures, urinary bladder replacement, urethra substitutes and cellular therapies for the treatment of urinary incontinence. The Tissue Engineering approach has major advantages over traditional organ transplantation and circumvents the problem of organ shortage. Tissues reconstructed from readily available biopsy material induce only minimal or no immunogenicity when reimplanted in the patient. This book is aimed at anyone interested in the application of Tissue Engineering in different organ systems. It offers insights into a wide variety of strategies applying the principles of Tissue Engineering to tissue and organ regeneration.

\title{
How to reference
}

In order to correctly reference this scholarly work, feel free to copy and paste the following:

Nithya Joseph, Anil Kumar P R and TV Kumary (2011). Tunable Stimuli-Responsive Polymers for Cell Sheet Engineering, Regenerative Medicine and Tissue Engineering - Cells and Biomaterials, Prof. Daniel Eberli (Ed.), ISBN: 978-953-307-663-8, InTech, Available from: http://www.intechopen.com/books/regenerative-medicineand-tissue-engineering-cells-and-biomaterials/tunable-stimuli-responsive-polymers-for-cell-sheet-engineering

\section{INTECH}

open science | open minds

\author{
InTech Europe \\ University Campus STeP Ri \\ Slavka Krautzeka 83/A \\ 51000 Rijeka, Croatia \\ Phone: +385 (51) 770447 \\ Fax: +385 (51) 686166 \\ www.intechopen.com
}

\author{
InTech China \\ Unit 405, Office Block, Hotel Equatorial Shanghai \\ No.65, Yan An Road (West), Shanghai, 200040, China \\ 中国上海市延安西路65号上海国际贵都大饭店办公楼 405 单元 \\ Phone: +86-21-62489820 \\ Fax: $+86-21-62489821$
}


(C) 2011 The Author(s). Licensee IntechOpen. This chapter is distributed under the terms of the Creative Commons Attribution-NonCommercialShareAlike-3.0 License, which permits use, distribution and reproduction for non-commercial purposes, provided the original is properly cited and derivative works building on this content are distributed under the same license. 